\title{
A unified picture for three different cosmic-ray observables
}

\section{Ottavio Fornieri, ${ }^{a}$ Daniele Gaggero, ${ }^{b, *}$ Daniel Guberman, ${ }^{c}$ Loann Brahimi, ${ }^{d}$ Pedro De La Torre Luque ${ }^{e}$ and Alexandre Marcowith ${ }^{d}$}

\author{
${ }^{a}$ Deutsches Elektronen-Synchrotron (DESY), Platanenallee 6, D-15738 Zeuthen, Germany \\ ${ }^{b}$ Instituto de Física Teórica UAM-CSIC, Campus de Cantoblanco, E-28049 Madrid, Spain \\ ${ }^{c}$ Department of Physical Sciences, Earth and Environment, University of Siena, Strada Laterina 8, 53100 \\ Siena, Italy \\ ${ }^{d}$ Laboratoire Universe et Particules de Montpellier (LUPM) Un. Montpellier, CNRS IN2P3, CC72, place \\ E. Bataillon, 34095, Montpellier Cedex 5, France \\ ${ }^{e}$ The Oskar Klein Centre, Department of Physics, Stockholm University, AlbaNova SE-10691 Stockholm, \\ Sweden \\ E-mail: ottavio.fornieri@desy.de, daniele.gaggero@uam.es
}

\begin{abstract}
We present here a unified scenario that connects three peculiar spectral features recently reported in the spectra of charged cosmic rays (CRs). The spectral hardening measured by AMS-02 in the hadronic spectra above $\sim 250 \mathrm{GeV}$ is here interpreted as a diffusion imprint, and modeled by means of a transport coefficient that smoothly hardens with rigidity. We implement such propagation framework to solve the transport equation with the DRAGON2 numerical code in order to determine the large-scale contribution to the CR fluxes. On top of this solution we explore the hypothesis of a nearby, hidden Supernova Remnant (SNR) to be responsible for the high-energy (above $\sim 100 \mathrm{GeV}$ ) all-lepton flux, in particular for the spectral break consistently measured by all the space- and ground-based detectors around $1 \mathrm{TeV}$. We compute such contribution analytically adopting the same propagation setup implemented for the large-scale background. Simultaneously, we find the signature of the same source in the peculiar bump structure observed by the DAMPE Collaboration in the proton spectrum, consisting of a strong hardening at $\sim 500 \mathrm{GeV}$ and a softening at $13 \mathrm{TeV}$. We validate our hypothesis with the CR dipole-anisotropy (DA) amplitude and phase. In particular, we interpret the high-energy data (above $10 \mathrm{TeV}$ ) pointing towards the Galactic Center as the convolution of the directional fluxes of the large-scale-background sources, whereas the DA amplitude below that energy is compatible with the predictions of our model and is therefore considered as a signature of the nearby SNR that we invoke.
\end{abstract}

$37^{\text {th }}$ International Cosmic Ray Conference (ICRC 2021)

July 12th - 23rd, 2021

Online - Berlin, Germany

\footnotetext{
*Presenter
} 


\section{Introduction}

A dramatic improvement in the accuracy of both hadronic and leptonic cosmic-ray (CR) measurements has been recently achieved. This progress offered a unique opportunity to shed light on several key questions concerning the acceleration and transport mechanisms of the non-thermal cosmic particles that permeate our Galaxy [1]. In particular, the AMS-02 Collaboration recently reported the absolute fluxes of light nuclear species [2,3] and pointed out a progressive spectral hardening at high rigidities $(\sim 250 \mathrm{GV})$. This feature is twice as large in the secondary species [4], suggesting a diffusive origin [5]. Even more recently, the DAMPE experiment confirmed this spectral break in the high-energy proton spectrum and also observed a softening at $13.6 \mathrm{TeV}$, characterized by a change in the spectral index from 2.60 to 2.85 [6]. Such structured feature may be the signature of a nearby CR accelerator. As shown in [7], this hypothetical source could only be simultaneously compatible with the observed CR flux and the measured dipole anisotropy if the diffusion of the particles leaving the source is very slow.

Another attempt to simultaneously reproduce the nuclei spectra and the dipole anisotropy recently considered a two-zone (disk/halo) diffusion, though applied to the background particles only and with no connection with the leptonic spectrum [8,9].

The H.E.S.S. [10, 11], CALET [12] and DAMPE [13] collaborations have also measured a break at $1 \mathrm{TeV}$ in the all-electron spectrum, which could be a signature of a local CR electron source. As first shown in [14] and further discussed in [15], this source should not produce electrons and positrons in similar amounts in order to be compatible with the observed positron fraction: a nearby Supernova Remnant (SNR) would hence fulfill this requirements. Among the known nearby SNRs, Vela and Cygnus Loop appeared as promising candidates due to their age and distance, but recent studies have shown that their expected contribution to the all-electron flux at high-energy $(E>1 \mathrm{TeV}$ ) would be sub-dominant (see for example [16]).

Here we propose a novel, comprehensive scenario that simultaneously explains the bump in the CR spectrum, the all-electron spectrum and the dipole anisotropy. This model is based on the following arguments: (i) We argue that a nearby fading SNR is associated to both the hadronic bump measured by DAMPE and the break in the all-lepton spectrum at $1 \mathrm{TeV}$ outlined by H.E.S.S. (ii) We adopt a transport scenario featuring a rigidity scaling that progressively hardens with increasing energy, motivated by AMS-02 data. We demonstrate that this is essential to satisfy the constraints imposed by the measured anisotropy.

\section{The transport setup}

In this section we describe the propagation setup that will be used throughout the paper. We refer to a forthcoming publication [17] (hereafter F21b) for a more extensive description. We consider two distinct populations of cosmic particles: (i) a diffuse large-scale background of hadronic and leptonic species, (ii) an extra contribution from a nearby accelerator. The propagation of the first population is computed by solving the general diffusion-loss transport equation with the DRAGON ${ }^{1}$ package.

\footnotetext{
1DRAGON (https://github.com/cosmicrays/DRAGON) [18] takes into account all the physical processes from low-energy up to high-energy effects, and the physical parameters implemented in our run are the same described in [15].
} 
We calculate the contribution from the second population in a semi-analytical way, by solving the transport equation written in the following general form:

$$
\frac{\partial f(E, t, r)}{\partial t}=\frac{D(E)}{r^{2}} \frac{\partial}{\partial r} r^{2} \frac{\partial f}{\partial r}+\frac{\partial}{\partial E}(b(E) f)+Q(E, t, r),
$$

where $Q(E, t, r)=S(E) L(t) \delta(r)$ considers a time-dependent injection, $D(E)=D_{0}\left(\frac{E}{E_{0}}\right)^{\delta(E)}$ and the loss term, $b(E)=-\frac{4}{3} c \sigma_{T}\left[f_{\mathrm{KN}}^{i} U_{i}+U_{B}\right]\left(\frac{E}{m_{e} c^{2}}\right)^{2}$, includes the Klein-Nishina $(\mathrm{KN})$ relativistic correction to the Inverse Compton (IC) cross-section, as discussed in [19, 20]. The details of the calculation to solve Equation (1), as well as the expression for the $\mathrm{KN}$ correction factor, are presented in F21b.

The key difference with respect to previous studies resides in the transport setup, characterized by the rigidity-dependent diffusion coefficient $D(\rho)$ for both the smooth large-scale component and the extra contribution.

Motivated by the analyses on secondary/primary ratio measured by AMS-02, we adopt the phenomenological setup considered in [21]. In that scenario the diffusion coefficient slope, which is commonly parametrized as $D(E)=D_{0}\left(\frac{E}{E_{0}}\right)^{\delta(E)}$ (with $D_{0}$ normalization at reference energy $E_{0}$ and $\delta$ here changing with $E$ ), smoothly hardens as rigidity increases:

$$
\frac{d \log D(\rho)}{d \log \rho} \equiv \gamma(\rho) \approx \gamma_{\text {high }}+\frac{\Delta}{1+\frac{\xi}{1-\xi}\left(\frac{\rho}{\rho_{0}}\right)^{\Delta}},
$$

where $\rho=E / Z e$ is the rigidity of the particle of charge $\mathrm{Z}, \rho_{0}$ is a reference rigidity, and $\left(\gamma_{\text {high }}, \Delta, \xi\right)$ are free parameters.

Slightly modifying the THMb (Two-Halo Model b) model described in [21] for the updated datapoints, the reference values of the parameters are: $\gamma_{\text {high }}=0.19, \Delta=0.53, \xi=0.1$, with a normalized diffusion coefficient $D_{0}=1.21 \cdot 10^{28} \mathrm{~cm}^{2} \mathrm{~s}^{-1}$ at reference rigidity $2 \mathrm{GV}$. With these parameters, the diffusion coefficient scales as $\sim E^{0.67}$ in the lowest-energy region $(E \sim 1 \mathrm{GeV})$ and hardens up to $\sim E^{0.20}$ at the highest energies $\left(E>10^{4} \mathrm{GeV}\right)$, exhibiting a smooth transition.

The key point shown in [21] is that such a setup is formally equivalent to a two-zone transport model featuring a change in the properties of the interstellar medium (ISM) between an inner-halo $(|z|<\xi L)$ region and an extended-halo $(\xi L<|z|<L)$ region, where $L \sim 4 \mathrm{kpc}$ and $\xi \sim O(0.1)$ (see [22] for a discussion about the transport microphysics that can be associated to this class of models).

In this work, the transport setup described in Equation (2) is adopted consistently in both the large-scale propagation and in the propagation of particles from the nearby remnant. We will show that it plays a key role in reconciling the high-energy break in the all-lepton $\operatorname{spectrum}\left(E_{e^{ \pm}} \sim 1 \mathrm{TeV}\right)$ with the bump recently reported by DAMPE in the proton spectrum at $E_{p} \sim 10 \mathrm{TeV}$, correctly reproducing the CR anisotropy data.

\section{A consistent picture of electron, proton and anisotropy data}

As pointed out in the Introduction, we are assuming that the particles accelerated nearby can contribute to a large fraction of the observed high-energy lepton flux. Based on the rate of Supernova 
events in the Galaxy [23] and on the massive losses that leptons undergo during the journey towards the Earth, we find (see F21b) that it is reasonable to consider only one nearby SNR explosion. The accelerator we are considering is an old, hidden SNR characterized by a distance $d=300 \mathrm{pc}$ and an age $t_{\text {age }}=2 \cdot 10^{5} \mathrm{yr}$, injecting cosmic electrons and protons. Those values result from the constraints imposed by the break at $\sim 1 \mathrm{TeV}$ in the observed all-lepton flux, as it is discussed in detail in F21b. We assume that particles remain confined inside the SN shock as long as their energy is lower than a maximum escape energy $E_{\text {esc }}$. This implies an energy-dependent release time that is regulated by the different stages of the SNR evolution and is different for protons and electrons. A detailed calculation of these evolution stages, as well as of the required escape energy, is reported in F21b, where they are modelled based on a number of parameters characterizing the ISM. Such parameters are not the result of a fitting procedure, but rather they are chosen based on dedicated independent observations. After the escape, particles are injected into the ISM according to the luminosity function $L(t)=L_{0} /\left(1+\frac{t}{\tau_{\mathrm{d}}}\right)^{\alpha_{\mathrm{d}}}$ [24]. In this work, the best fit is obtained for $\tau_{\mathrm{d}}=10^{5} \mathrm{yr}, \alpha_{\mathrm{d}}=2$ which would correspond to fast escape (large $\delta$ values) in [25].

\subsection{Results: All-lepton spectrum}

In Figure 1 we show the $e^{+}+e^{-}$propagated spectrum plotted against data from AMS-02 [26], CALET [12] and H.E.S.S. [11].

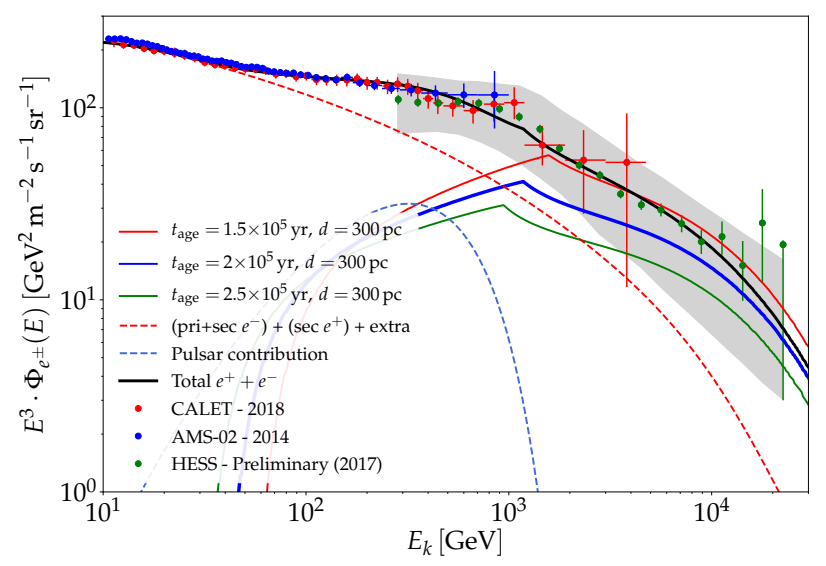

Figure 1: The all-lepton spectrum as the sum of a smooth background of primary $e^{-}+$secondary $e^{ \pm}+$extra $e^{ \pm}$(red dashed line), a fit of the positron flux (blue dashed line) and the single-source contribution calculated in this work for the corresponding age $t_{\mathrm{age}}=2 \cdot 10^{5} \mathrm{yr}$ (blue solid line). Other ages (red and green solid lines) are added for comparison.

The smooth background (red dashed line) is the sum of: (i) primary $e^{-}$, injected with DRAGON with a power-law spectrum $\Gamma_{\mathrm{inj}}^{\mathrm{DRA} e^{-}}=2.74$ and a cutoff $E_{\mathrm{cut}}^{\mathrm{DRA} e^{-}}=20 \mathrm{TeV}$ that is estimated equating the acceleration and loss timescales [27]; (ii) secondary $e^{ \pm}$, fixed by the DRAGON-propagated primary species; (iii) a smooth extra component of primary $e^{+}+e^{-}$pairs, that represents the convolution of a large $\left(O\left(10^{4}\right)\right)$ number of old $\left(t_{\mathrm{age}}>10^{6} \mathrm{yr}\right)$ pulsars (see [15]). The secondary leptons and the extra component contribute to the smooth background as $\sim 1 \%$ and $\sim 3 \%$, respectively. The blue dashed curve represents the total contribution from the class of sources generating lepton pairs (here we invoke pulsars). The three solid curves correspond to the contribution from the hidden 
remnant discussed in this work. They are computed by solving Equation (1) for different ages. The electron population is injected with a single power-law (slope $\Gamma_{\text {inj }}^{e^{-}}=2.45$ ). The difference with respect to the proton injection slope (see next section) is well justified by synchrotron losses that electrons undergo before being released [28]. The total energy budget associated to the leptonic population is $\simeq 4.5 \cdot 10^{47} \mathrm{erg}$, that, injected by a SNR with typical energy budget $E_{\mathrm{SNR}}=10^{51} \mathrm{erg}$, corresponds to an efficiency of conversion into leptons of $\sim 10^{-4}$, which is within the theoretical expectations [29]. Finally, the black curve is the sum of all the contributions, where we have chosen the source of age $t_{\text {age }}=2 \cdot 10^{5} \mathrm{yr}$ as reference (blue solid).

The plot shows how low-energy particles $(E \lesssim 100 \mathrm{GeV}$ ) are cut off as an effect of the energydependent release time, such that those particles did not have time to be released and propagate to the Earth.

As far as the luminosity function is concerned, we vary $\alpha_{\mathrm{d}} \in[1,3]$ and report negligible variations in the spectrum. On the other hand, while varying $\tau_{\mathrm{d}}$ in the range $\left[10^{4}, 2 \cdot 10^{5}\right] \mathrm{yr}$ does not qualitatively change the results, smaller values cannot reproduce the data points above the $\sim \mathrm{TeV}$ break. Indeed, since $\tau_{\mathrm{d}}$ acts as a timescale for the luminosity function, a quickly decaying luminosity would approach the limit of a burst-like injection $\left(L(t) \rightarrow L_{0} \delta\left(t-t_{\text {rel }}\right) d t\right)$, and accordingly the $\sim \mathrm{TeV}$ peak energy allowed by the source age would be followed by an abrupt cutoff in the spectrum. This leads us to conclude that a declining luminosity from the source is necessary to match the observations in the adopted assumption, i.e. with a unique hidden source.

\subsection{Results: Proton spectrum}

The proton data are characterized by a hardening at $\sim 200 \mathrm{GeV}$ and a softening at energies as high as $\sim 13 \mathrm{TeV}$. Here, we connect this feature to the same hidden remnant considered in the previous section.

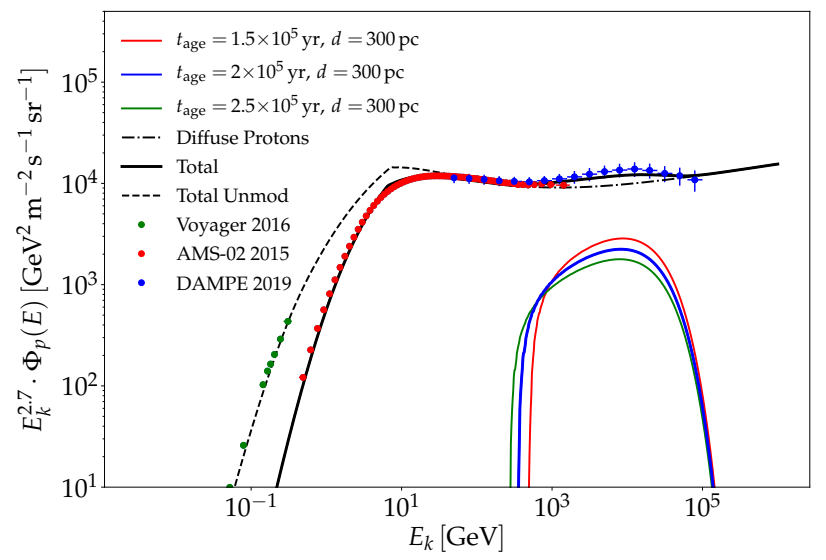

Figure 2: The protons spectrum, resulting from the sum of DRAGON modulated spectrum (black solid) and the solution of the single-source transport equation computed in this work for the age $t_{\mathrm{age}}=2 \cdot 10^{5} \mathrm{yr}$ (blue solid line). Other ages (red and green solid lines), as well as the unmodulated spectrum (black dashed line) are added for comparison.

In Figure 2, we show our result for different ages of the nearby remnant (green, red and blue solid lines). They were calculated assuming a power-law injection spectrum of $\Gamma_{\text {inj }}^{p}=2.1$, and 
an exponential high-energy cutoff at energy $E_{\text {cut }}=20 \mathrm{TeV}$, chosen to match the bump observed by DAMPE. Note that such high-energy cutoff is compatible with a maximum escape energy of $\sim \mathrm{PeV}$ considered at earlier stages $\left(\lesssim 10^{3} \mathrm{yr}\right)$ of the SNR. The normalization is consistent with a total hadronic energy budget $\simeq 2.5 \cdot 10^{49} \mathrm{erg}$, which corresponds to an efficiency of $\sim 10 \%$ in accelerating protons. Data points are from AMS-02 [30], Voyager [31] and DAMPE [6].

The smooth background is calculated using DRAGON with the same physical parameters dis-

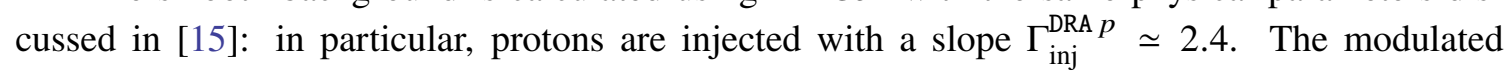
spectrum is computed with an effective potential $\left\langle\phi_{\text {mod }}\right\rangle=0.54$ [32]. The three solid lines are solutions of Equation (1) in the limit of negligible losses $\left(b\left(E_{\mathrm{t}}\right) \approx b(E) \rightarrow 0\right)$, shown for three different ages. The sum of the contributions is considered for the source of age $t_{\mathrm{age}}=2 \cdot 10^{5} \mathrm{yr}$. The total modulated (unmodulated) flux associated to this case is shown as a black solid (dashed) line.

As for the case of the all-lepton spectrum, the effect of the energy-dependent release cuts off the low-energy $(E \lesssim 100 \mathrm{GeV})$ part of the spectrum.

\subsection{CR dipole anisotropy}

The CR dipole anisotropy (DA) provides a crucial complimentary probe of model proposed in this work. The high degree of isotropy (up to 1 part in $\sim 10^{3}$ ) detected by a variety of experiments in a wide energy range is especially constraining as far as the contribution from a local source is concerned.

In this section we compute the predicted dipole anisotropy associated with the hidden remnant, following the formalism described in [17]. The interpretation of a single source as the origin of the spectral feature in the proton spectrum between $1 \mathrm{TeV}$ and $10 \mathrm{TeV}$ is heavily challenged in the context of a simple diffusion setup characterized by a single power-law. This consideration led the authors of several recent papers to consider more complex diffusion scenarios featuring a high-confinement zone near the source of interest (see for instance [7]).

Here, we consider instead the transport scenario directly suggested by the hardening in the light nuclei reported by the AMS-02 Collaboration, as described above.

In Figure 3, we show that the hypothesis of one nearby old remnant originating the CR populations responsible for both the leptonic and the hadronic features is compatible with the current anisotropy data. The dipole anisotropy is computed as the sum of two components: (i) the single-source contribution, that assumes directional observations; (ii) the averaged anisotropy of the large-scale background. The second component typically points towards the Galactic center and can be assumed to be a simple power-law [33]. We calculated this contribution using the fit parameters recently suggested in [7], according to which the background anisotropy can be written as $\Delta_{\mathrm{bkg}}=c_{1}\left(\frac{E}{1 \mathrm{PeV}}\right)^{c_{2}}$, where $\left(c_{1}, c_{2}\right)=\left(1.32 \cdot 10^{-3}, 0.62\right)$. The result is the green solid line in the figure. On the other hand, the single-source contribution is found under the assumption of diffusive behaviour for the released particles. This component corresponds to the red solid line in the figure, for the source of age $t_{\text {age }}=2 \cdot 10^{5} \mathrm{yr}$.

We highlight here that the dipole anisotropy shown in Figure 3 was obtained without any fine tuning of the source's parameters. It was indeed the result of the novel approach that we used to implement the diffusion coefficient for the single-source particles, which, according to Equation (2), has a harder slope at high energies $(\delta \lesssim 0.2$ at $E>10 \mathrm{TeV})$. 


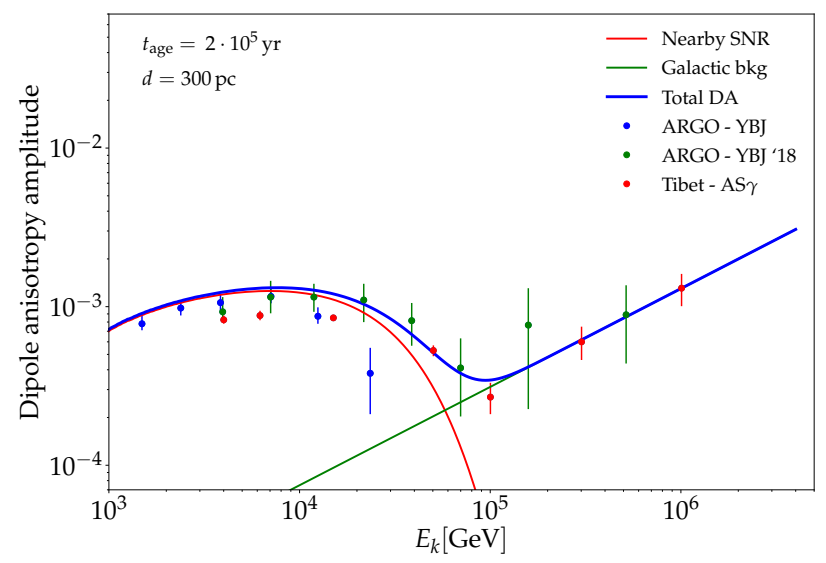

Figure 3: Cosmic-ray dipole anisotropy amplitude calculated as the sum of a background anisotropy (green solid line) and the single source contribution (red solid line) for the source of age $t_{\text {age }}=2 \cdot 10^{5} \mathrm{yr}$. Anisotropy data are consistent with each other, therefore here we plot a subset of them, to avoid confusion. For the plotted points are from ARGO and Tibet-AS $\gamma$ (see [33] and references therein.)

\section{Conclusions}

In this paper we proposed the idea that the spectral feature at $\sim 10 \mathrm{TeV}$ in the cosmic-ray proton spectrum recently reported by the DAMPE Collaboration together with the spectral break at $\sim 1 \mathrm{TeV}$ measured by H.E.S.S. in the lepton spectrum have a common origin and can be associated to a nearby, fading SNR. We believe this simultaneous interpretation to be of paramount importance, since SNRs are accelerators for both electrons and protons.

We computed the propagation of cosmic particles from such object in a spherically symmetric setup and assuming a luminosity that declines over time, and found that the most relevant observables can be simultaneously reproduced. The key ingredient in the calculation is a transport setup based on a diffusion coefficient characterized by a smooth transition to a progressively harder rigidity-scaling at higher energies, as suggested by the light nuclei spectra measured by the AMS-02 Collaboration. This feature allowed us to reproduce the cosmic-ray anisotropy data without any further assumptions. Moreover, the combined leptonic and hadronic data led us to characterize the properties of the particles accelerated by such object in good agreement with theoretical expectations.

\section{References}

[1] S. Gabici et al., Int. J. Mod. Phys. D Vol. 28, No. 15, 1930022 (2019).

[2] M. Aguilar et al., Phys. Rev. Lett. 114, 171103 (2015).

[3] M. Aguilar et al., Phys. Rev. Lett. 117, 231102 (2016).

[4] M. Aguilar et al., Phys. Rev. Lett. 120, 021101 (2018).

[5] Y. Genolini et al., Phys. Rev. Lett. 119241101 (2017).

[6] Q. An et al., Sci. Adv. Vol. 5 No. 9 (2019). 
[7] K. Fang et al., ApJ 90369 (2020).

[8] W. Liu et al., JCAP 10010 (2019).

[9] Q. Yuan et al., Front. Phys. (Beijing) Vol. 16 No. 2 (2021).

[10] F. Aharonian et al., A\&A Vol. 508 No. 2 (2009).

[11] D. Kerszberg, ICRC (2017).

[12] O. Adriani et al., Phys. Rev. Lett. 12026 (2018).

[13] G. Ambrosi et al., Nature 552 63-66 (2017).

[14] S. Recchia et al., Phys. Rev. D 9910 (2019).

[15] O. Fornieri et al., JCAP 02009 (2020).

[16] S. Manconi et al., JCAP 04024 (2019).

[17] O. Fornieri et al., Submitted to PRD.

[18] C. Evoli et al., JCAP 02 015, (2017).

[19] D. Hooper et al., Phys. Rev. D 96 10, (2017).

[20] C. Evoli et al., Phys. Rev. Lett. 126249901 (2021).

[21] N. Tomassetti, ApJ Lett. 752 L13 (2012).

[22] O. Fornieri et al., MNRAS 502, 5821-5838 (2021).

[23] K. Ferriere et al., Rev. Mod. Phys. Vol. 73 (2001).

[24] A. M. Atoyan et al., Phys. Rev. D 526 (1995).

[25] S. Celli et al., MNRAS 490, 4317-4333 (2019).

[26] M. Aguilar et al., Phys. Rev. Lett. 113221102 (2014).

[27] J. Vink, Astron. Astrophys. Rev. Vol. 201 (2012).

[28] R. Diesing et al., Phys. Rev. Lett. 123071101 (2019).

[29] V. Tatischeff, A\&A Vol. 499191 (2009).

[30] M. Aguilar et al., Phys. Rev. Lett. 114171103 (2015).

[31] A. C. Cummings et al., ApJ Vol. 831 No. 1 (2016).

[32] I. G. Usoskin et al., Journal of Geophysical Research (Space Physics) Vol. 116 (2011).

[33] M. Ahlers et al., Prog. Part. Nucl. Phys. Vol. 94 184-216 (2017). 